\title{
Supporting information for: Potential Energy Landscape of the Photoinduced Multiple Proton-Transfer Process in the Green Fluorescent Protein: Classical Molecular Dynamics and Multiconfigurational Electronic Structure Calculations
}

\author{
Oriol Vendrell, Ricard Gelabert*, Miquel Moreno and José M. Lluch
}

\section{Complete List-of-Authors for References 35 and 40}

35. MacKerell Jr., A. D.; Bashford, D.; Bellott, M.; Jr., R. L. D.; Evanseck, J. D.; Field, M. J.; Fischer, S.; Gao, J.; Guo, H.; Ha, S.; Joseph-McCarthy, D.; Kuchnir, L.; Kuczera, K.; Lau, F. T. K.; Mattos, C.; Michnick, S.; Ngo, T.; Nguyen, D. T.; Prodhom, B.; III, W. E. R.; Roux, B.; Schlenkrich, M.; Smith, J. C.; Stote, R.; Straub, J.; Watanabe, M.; Wiórkiewicz-Kuczera, J.; Yin, D.; Karplus, M. J. Phys. Chem. B 1998, 102, 3586-3616.

40. Andersson, K.; Barysz, M.; Bernhardsson, A.; Blomberg, M. R. A.; Carissan, Y.; Cooper, D. L.; Fülscher, M. P.; Gagliardi, L.; de Graaf, C.; Hess, B. A.; Karlström, G.; Lindh, R.; Malmqvist, P.-Å.; Nakajima, T.; Neogrády, P.; Olsen, J.; Roos, B. O.; Schimmelpfennig, B.; Schüz, M.; Seijo, L.; Serrano-Andrés, L.; Siegbahn, P. E. M.; Stålring, J.; Thorsteinsson, T.; Veryazov, V.; Widmark, P.-O. "MOLCAS 6.0: User's Guide", 2004.

\section{Proton transfer profiles}

All the calculated proton transfer energy profiles are included for the $\mathrm{S}_{0},{ }^{1} \pi \pi^{*}$ and ${ }^{1} \pi \sigma^{*}$ electronic states. $r_{i}$ is the coordinate representing the position of each proton, while the $R_{i}$ coordinates represent the oxygen-oxygen distances (see text). Distance values are in $\AA$. The rightmost column contains the profile identification in terms of the two involved protonation states and the oxygen-oxygen distance being scanned. Energy values in kcal $/ \mathrm{mol}$, relative to the minimum energy structure in $\mathrm{S}_{0}$, are shown for the CASSCF and CASPT2 levels of theory with the $(6,6)$ active space

\begin{tabular}{ccccccccccccc}
\hline & \multicolumn{1}{c}{} & \multicolumn{1}{c}{ CASSCF } & \multicolumn{3}{c}{ CASPT2 } \\
\hline$r_{1}$ & $r_{2}$ & $r_{3}$ & $R_{1}$ & $R_{2}$ & $R_{3}$ & $\mathrm{~S}_{0}$ & ${ }^{1} \pi \pi^{*}$ & ${ }^{1} \pi \sigma^{*}$ & $\mathrm{~S}_{0}$ & ${ }^{1} \pi \pi^{*}$ & ${ }^{1} \pi \sigma^{*}$ & prof. $^{1}$ \\
\hline-0.3300 & -0.2900 & -0.2500 & 2.6600 & 2.5800 & 2.3000 & 9.532 & 115.190 & 144.574 & 11.067 & 95.851 & 131.121 & CWS-CWG-da-2.300 \\
-0.3300 & -0.2900 & -0.1667 & 2.6600 & 2.5800 & 2.3000 & 4.440 & 109.902 & 140.820 & 4.775 & 89.324 & 126.070 & CWS-CWG-da-2.300 \\
-0.3300 & -0.2900 & -0.0833 & 2.6600 & 2.5800 & 2.3000 & 4.840 & 110.065 & 142.767 & 4.297 & 88.565 & 127.004 & CWS-CWG-da-2.300 \\
-0.3300 & -0.2900 & -0.0000 & 2.6600 & 2.5800 & 2.3000 & 7.141 & 112.098 & 146.751 & 6.282 & 90.234 & 130.515 & CWS-CWG-da-2.300 \\
-0.3300 & -0.2900 & 0.0833 & 2.6600 & 2.5800 & 2.3000 & 10.003 & 114.679 & 151.309 & 9.525 & 93.149 & 135.292 & CWS-CWG-da-2.300 \\
-0.3300 & -0.2900 & 0.1667 & 2.6600 & 2.5800 & 2.3000 & 14.282 & 118.686 & 157.160 & 14.807 & 98.110 & 142.016 & CWS-CWG-da-2.300
\end{tabular}




\begin{tabular}{|c|c|c|c|c|c|c|c|c|c|c|c|c|}
\hline-0.3300 & -0.2900 & 0.2500 & 2.6600 & 2.5800 & 2.3000 & 23.244 & 127.403 & 167.477 & 25.156 & 108.167 & 153.627 & CWS-CWG-da- 2.300 \\
\hline-0.3274 & -0.2937 & -0.3380 & 2.6548 & 2.5874 & 2.4759 & 4.587 & 110.791 & 137.967 & 6.375 & 91.702 & 124.786 & CWS-CWG-da- 2.476 \\
\hline-0.3274 & -0.2937 & -0.2253 & 2.6548 & 2.5874 & 2.4759 & 0.000 & 105.971 & 134.921 & 0.000 & 85.047 & 119.839 & CWS-CWG-da- 2.476 \\
\hline-0.3274 & -0.2937 & -0.1127 & 2.6548 & 2.5874 & 2.4759 & 3.649 & 109.312 & 140.480 & 2.219 & 86.903 & 123.803 & CWS-CWG-da- 2.476 \\
\hline-0.3274 & -0.2937 & -0.0000 & 2.6548 & 2.5874 & 2.4759 & 7.957 & 113.250 & 146.981 & 5.962 & 90.218 & 129.522 & CWS-CWG-da- 2.476 \\
\hline-0.3274 & -0.2937 & 0.1127 & 2.6548 & 2.5874 & 2.4759 & 10.186 & 115.085 & 151.440 & 8.811 & 92.611 & 134.383 & CWS-CWG-da- 2.476 \\
\hline-0.3274 & -0.2937 & 0.2253 & 2.6548 & 2.5874 & 2.4759 & 12.130 & 116.661 & 155.373 & 12.353 & 95.723 & 139.766 & CWS-CWG-da- 2.476 \\
\hline-0.3300 & -0.2900 & -0.4250 & 2.6600 & 2.5800 & 2.6500 & 3.925 & 110.683 & 136.208 & 5.875 & 91.744 & 123.189 & CWS-CWG-da- 2.650 \\
\hline-0.3300 & -0.2900 & -0.2833 & 2.6600 & 2.5800 & 2.6500 & 1.217 & 107.718 & 135.171 & 0.740 & 86.298 & 119.600 & CWS-CWG-da- 2.650 \\
\hline-0.3300 & -0.2900 & -0.1417 & 2.6600 & 2.5800 & 2.6500 & 9.473 & 115.604 & 145.629 & 6.879 & 92.001 & 127.744 & CWS-CWG-da-2.650 \\
\hline-0.3300 & -0.2900 & 0.0000 & 2.6600 & 2.5800 & 2.6500 & 16.237 & 121.895 & 155.056 & 12.722 & 97.302 & 135.963 & CWS-CWG-da-2.650 \\
\hline-0.3300 & -0.2900 & 0.1417 & 2.6600 & 2.5800 & 2.6500 & 17.184 & 122.330 & 158.754 & 14.603 & 98.603 & 140.318 & CWS-CWG-da-2.650 \\
\hline-0.3300 & -0.2900 & 0.2833 & 2.6600 & 2.5800 & 2.6500 & 15.146 & 119.826 & 159.078 & 14.900 & 98.363 & 142.833 & CWS-CWG-da- 2.650 \\
\hline-0.3300 & -0.2900 & 0.4250 & 2.6600 & 2.5800 & 2.6500 & 22.010 & 126.314 & 113.188 & 24.497 & 107.520 & 121.314 & CWS-CWG-da- 2.650 \\
\hline-0.3300 & -0.2500 & -0.2380 & 2.6600 & 2.3000 & 2.4759 & 7.305 & 115.664 & 143.400 & 12.838 & 96.154 & 130.916 & CWS-CSS-da-2.300 \\
\hline-0.3300 & -0.1667 & -0.2380 & 2.6600 & 2.3000 & 2.4759 & 5.840 & 110.440 & 140.596 & 4.967 & 88.695 & 124.865 & CWS-CSS-da-2.300 \\
\hline-0.3300 & -0.0833 & -0.2380 & 2.6600 & 2.3000 & 2.4759 & 7.646 & 111.656 & 143.270 & 5.055 & 88.171 & 125.629 & CWS-CSS-da- 2.300 \\
\hline-0.3300 & 0.0000 & -0.2380 & 2.6600 & 2.3000 & 2.4759 & 11.605 & 114.953 & 148.128 & 7.720 & 90.154 & 128.953 & CWS-CSS-da-2.300 \\
\hline-0.3300 & 0.0833 & -0.2380 & 2.6600 & 2.3000 & 2.4759 & 16.244 & 118.907 & 153.618 & 11.712 & 93.437 & 133.585 & CWS-CSS-da- 2.300 \\
\hline-0.3300 & 0.1667 & -0.2380 & 2.6600 & 2.3000 & 2.4759 & 17.630 & 124.274 & 160.361 & 20.324 & 98.805 & 140.114 & CWS-CSS-da- 2.300 \\
\hline-0.3300 & 0.2500 & -0.2380 & 2.6600 & 2.3000 & 2.4759 & 28.121 & 134.247 & 171.470 & 31.410 & 109.292 & 151.549 & CWS-CSS-da- 2.300 \\
\hline-0.3300 & -0.3250 & -0.2379 & 2.6600 & 2.4500 & 2.4759 & 4.844 & 110.694 & 138.937 & 6.626 & 91.601 & 125.916 & CWS-CSS-da- 2.450 \\
\hline-0.3300 & -0.2167 & -0.2379 & 2.6600 & 2.4500 & 2.4759 & 1.140 & 106.440 & 136.136 & 0.519 & 84.922 & 120.533 & CWS-CSS-da- 2.450 \\
\hline-0.3300 & -0.1083 & -0.2379 & 2.6600 & 2.4500 & 2.4759 & 5.786 & 110.379 & 141.860 & 2.844 & 86.520 & 123.709 & CWS-CSS-da- 2.450 \\
\hline-0.3300 & -0.0000 & -0.2379 & 2.6600 & 2.4500 & 2.4759 & 11.796 & 115.553 & 149.043 & 7.098 & 89.924 & 128.847 & CWS-CSS-da- 2.450 \\
\hline-0.3300 & 0.2167 & -0.2379 & 2.6600 & 2.4500 & 2.4759 & 16.443 & 123.104 & 160.304 & 18.468 & 96.981 & 139.100 & CWS-CSS-da- 2.450 \\
\hline-0.3300 & 0.3250 & -0.2379 & 2.6600 & 2.4500 & 2.4759 & 27.236 & 133.671 & 171.790 & 30.301 & 104.202 & 151.319 & CWS-CSS-da-2.450 \\
\hline-0.3274 & -0.3937 & -0.2379 & 2.6548 & 2.5874 & 2.4759 & 3.416 & 109.843 & 137.431 & 5.790 & 91.312 & 124.896 & CWS-CSS-da- 2.587 \\
\hline-0.3274 & -0.2625 & -0.2379 & 2.6548 & 2.5874 & 2.4759 & 0.847 & 106.692 & 135.829 & 0.330 & 85.250 & 120.193 & CWS-CSS-da- 2.587 \\
\hline-0.3274 & -0.1312 & -0.2379 & 2.6548 & 2.5874 & 2.4759 & 8.983 & 114.033 & 145.191 & 5.551 & 89.661 & 126.379 & CWS-CSS-da- 2.587 \\
\hline-0.3274 & 0.0000 & -0.2379 & 2.6548 & 2.5874 & 2.4759 & 17.151 & 121.214 & 154.765 & 11.506 & 94.621 & 133.404 & CWS-CSS-da-2.587 \\
\hline-0.3274 & 0.1312 & -0.2379 & 2.6548 & 2.5874 & 2.4759 & 21.293 & 124.298 & 160.258 & 14.880 & 96.922 & 137.797 & CWS-CSS-da- 2.587 \\
\hline-0.3274 & 0.2625 & -0.2379 & 2.6548 & 2.5874 & 2.4759 & 18.881 & 125.499 & 163.462 & 20.276 & 98.751 & 141.355 & CWS-CSS-da- 2.587 \\
\hline-0.3274 & 0.3937 & -0.2379 & 2.6548 & 2.5874 & 2.4759 & 28.813 & 135.102 & 174.119 & 31.719 & 105.457 & 153.201 & CWS-CSS-da- 2.587 \\
\hline-0.2500 & -0.2937 & -0.2379 & 2.3000 & 2.5874 & 2.4759 & 7.382 & 115.476 & 138.005 & 12.995 & 96.205 & 125.744 & CWS-WWS-da- 2.300 \\
\hline-0.1667 & -0.2937 & -0.2379 & 2.3000 & 2.5874 & 2.4759 & 7.978 & 111.074 & 130.558 & 7.448 & 89.698 & 114.661 & CWS-WWS-da- 2.300 \\
\hline-0.0833 & -0.2937 & -0.2379 & 2.3000 & 2.5874 & 2.4759 & 12.096 & 113.687 & 127.557 & 10.192 & 86.251 & 109.517 & CWS-WWS-da- 2.300 \\
\hline 0.0000 & -0.2937 & -0.2379 & 2.3000 & 2.5874 & 2.4759 & 13.806 & 117.748 & 126.000 & 18.281 & 89.102 & 106.240 & CWS-WWS-da- 2.300 \\
\hline 0.0833 & -0.2937 & -0.2379 & 2.3000 & 2.5874 & 2.4759 & 20.844 & 122.445 & 124.782 & 25.221 & 93.256 & 103.900 & CWS-WWS-da-2.300 \\
\hline 0.1667 & -0.2937 & -0.2379 & 2.3000 & 2.5874 & 2.4759 & 29.130 & 128.490 & 124.320 & 34.086 & 99.432 & 110.211 & CWS-WWS-da-2.300 \\
\hline 0.2500 & -0.2937 & -0.2379 & 2.3000 & 2.5874 & 2.4759 & 41.682 & 139.061 & 127.693 & 47.687 & 110.631 & 117.559 & CWS-WWS-da- 2.300 \\
\hline-0.3500 & -0.2937 & -0.2380 & 2.5000 & 2.5874 & 2.4759 & 2.943 & 109.318 & 138.220 & 4.748 & 90.407 & 125.563 & CWS-WWS-da-2.500 \\
\hline-0.2333 & -0.2937 & -0.2380 & 2.5000 & 2.5874 & 2.4759 & 1.984 & 106.431 & 130.349 & 1.453 & 84.971 & 114.513 & CWS-WWS-da-2.500 \\
\hline-0.1167 & -0.2937 & -0.2380 & 2.5000 & 2.5874 & 2.4759 & 10.716 & 113.209 & 130.142 & 8.044 & 84.877 & 111.189 & CWS-WWS-da-2.500 \\
\hline 0.0000 & -0.2937 & -0.2380 & 2.5000 & 2.5874 & 2.4759 & 16.093 & 120.105 & 129.444 & 19.231 & 89.953 & 108.041 & CWS-WWS-da-2.500 \\
\hline 0.1167 & -0.2937 & -0.2380 & 2.5000 & 2.5874 & 2.4759 & 23.777 & 124.605 & 125.809 & 26.894 & 93.851 & 103.057 & CWS-WWS-da- 2.500 \\
\hline 0.2333 & -0.2937 & -0.2380 & 2.5000 & 2.5874 & 2.4759 & 30.470 & 128.347 & 119.368 & 34.747 & 98.175 & 107.404 & CWS-WWS-da-2.500 \\
\hline 0.3500 & -0.2937 & -0.2380 & 2.5000 & 2.5874 & 2.4759 & 48.872 & 138.904 & 121.363 & 47.087 & 109.980 & 113.296 & CWS-WWS-da-2.500 \\
\hline-0.4274 & -0.2937 & -0.2379 & 2.6548 & 2.5874 & 2.4759 & 1.448 & 108.822 & 141.092 & 3.688 & 90.307 & 128.885 & CWS-WWS-da-2.655 \\
\hline-0.2849 & -0.2937 & -0.2379 & 2.6548 & 2.5874 & 2.4759 & 2.316 & 107.652 & 134.599 & 1.620 & 85.959 & 118.532 & CWS-WWS-da-2.655 \\
\hline-0.1425 & -0.2937 & -0.2379 & 2.6548 & 2.5874 & 2.4759 & 11.817 & 119.418 & 137.416 & 14.244 & 94.730 & 118.824 & CWS-WWS-da-2.655 \\
\hline 0.0000 & -0.2937 & -0.2379 & 2.6548 & 2.5874 & 2.4759 & 24.149 & 128.240 & 138.274 & 25.927 & 96.598 & 115.184 & CWS-WWS-da-2.655 \\
\hline 0.1425 & -0.2937 & -0.2379 & 2.6548 & 2.5874 & 2.4759 & 31.697 & 131.973 & 131.570 & 33.601 & 99.793 & 114.452 & CWS-WWS-da-2.655 \\
\hline 0.2849 & -0.2937 & -0.2379 & 2.6548 & 2.5874 & 2.4759 & 35.583 & 132.407 & 119.941 & 39.265 & 101.349 & 108.225 & CWS-WWS-da- 2.655 \\
\hline 0.4274 & -0.2937 & -0.2379 & 2.6548 & 2.5874 & 2.4759 & 47.236 & 144.906 & 119.848 & 53.281 & 116.679 & 113.207 & CWS-WWS-da-2.655 \\
\hline-0.3300 & -0.2500 & 0.2379 & 2.6600 & 2.3000 & 2.4759 & 22.244 & 125.930 & 165.607 & 23.645 & 106.199 & 151.356 & CWG-CSG-da-2.300 \\
\hline-0.3300 & -0.1667 & 0.2379 & 2.6600 & 2.3000 & 2.4759 & 15.685 & 118.828 & 159.890 & 15.309 & 97.279 & 143.663 & CWG-CSG-da-2.300 \\
\hline-0.3300 & -0.0833 & 0.2379 & 2.6600 & 2.3000 & 2.4759 & 9.866 & 116.931 & 159.553 & 15.028 & 93.772 & 141.492 & CWG-CSG-da- 2.300 \\
\hline-0.3300 & 0.0000 & 0.2379 & 2.6600 & 2.3000 & 2.4759 & 10.314 & 116.744 & 161.001 & 14.441 & 92.467 & 141.622 & CWG-CSG-da- 2.300 \\
\hline-0.3300 & 0.0833 & 0.2379 & 2.6600 & 2.3000 & 2.4759 & 11.304 & 119.433 & 162.923 & 15.075 & 91.499 & 142.842 & CWG-CSG-da-2.300 \\
\hline-0.3300 & 0.1667 & 0.2379 & 2.6600 & 2.3000 & 2.4759 & 13.833 & 121.359 & 166.244 & 17.902 & 93.653 & 146.117 & CWG-CSG-da-2.300 \\
\hline-0.3300 & 0.2500 & 0.2379 & 2.6600 & 2.3000 & 2.4759 & 21.296 & 126.227 & 174.327 & 26.119 & 98.121 & 154.638 & CWG-CSG-da-2.300 \\
\hline-0.3300 & -0.3250 & 0.2379 & 2.6600 & 2.4500 & 2.4759 & 18.603 & 123.022 & 161.671 & 20.690 & 103.972 & 148.114 & CWG-CSG-da- 2.450 \\
\hline-0.3300 & -0.2167 & 0.2379 & 2.6600 & 2.4500 & 2.4759 & 12.101 & 115.904 & 156.167 & 11.864 & 94.496 & 140.062 & CWG-CSG-da- 2.450 \\
\hline-0.3300 & -0.1083 & 0.2379 & 2.6600 & 2.4500 & 2.4759 & 13.117 & 116.145 & 158.351 & 10.722 & 92.538 & 139.792 & CWG-CSG-da- 2.450 \\
\hline-0.3300 & -0.0000 & 0.2379 & 2.6600 & 2.4500 & 2.4759 & 10.253 & 116.964 & 161.288 & 13.500 & 91.856 & 140.942 & CWG-CSG-da- 2.450 \\
\hline-0.3300 & 0.1083 & 0.2379 & 2.6600 & 2.4500 & 2.4759 & 10.262 & 116.492 & 162.511 & 13.182 & 86.702 & 141.398 & CWG-CSG-da-2.450 \\
\hline-0.3300 & 0.2167 & 0.2379 & 2.6600 & 2.4500 & 2.4759 & 15.293 & 118.061 & 163.798 & 11.645 & 89.903 & 142.928 & CWG-CSG-da-2.450 \\
\hline-0.3300 & 0.3250 & 0.2379 & 2.6600 & 2.4500 & 2.4759 & 17.852 & 122.659 & 171.766 & 22.667 & 94.545 & 151.759 & CWG-CSG-da- 2.450 \\
\hline-0.3274 & -0.2625 & 0.2379 & 2.6548 & 2.5874 & 2.4759 & 12.731 & 117.045 & 156.459 & 12.479 & 95.615 & 140.301 & CWG-CSG-da-2.587 \\
\hline-0.3274 & -0.1312 & 0.2379 & 2.6548 & 2.5874 & 2.4759 & 16.834 & 120.263 & 161.924 & 13.824 & 96.031 & 142.677 & CWG-CSG-da-2.587 \\
\hline-0.3274 & 0.0000 & 0.2379 & 2.6548 & 2.5874 & 2.4759 & 15.454 & 122.356 & 166.564 & 17.656 & 96.236 & 145.068 & CWG-CSG-da- 2.587 \\
\hline-0.3274 & 0.1312 & 0.2379 & 2.6548 & 2.5874 & 2.4759 & 14.127 & 122.375 & 166.703 & 16.106 & 92.731 & 144.427 & CWG-CSG-da- 2.587 \\
\hline-0.3274 & 0.2625 & 0.2379 & 2.6548 & 2.5874 & 2.4759 & 16.036 & 116.672 & 164.944 & 11.911 & 86.945 & 144.763 & CWG-CSG-da-2.587 \\
\hline-0.3274 & 0.3937 & 0.2379 & 2.6548 & 2.5874 & 2.4759 & 17.418 & 122.071 & 171.831 & 22.231 & 93.949 & 151.548 & CWG-CSG-da-2.587 \\
\hline-0.2500 & -0.2937 & 0.2379 & 2.3000 & 2.5874 & 2.4759 & 22.403 & 125.525 & 159.777 & 23.700 & 105.819 & 145.617 & CWG-WWG-da-2.300 \\
\hline-0.1667 & -0.2937 & 0.2379 & 2.3000 & 2.5874 & 2.4759 & 15.055 & 121.441 & 151.056 & 21.875 & 95.572 & 134.531 & CWG-WWG-da-2.300 \\
\hline-0.0833 & -0.2937 & 0.2379 & 2.3000 & 2.5874 & 2.4759 & 18.080 & 122.380 & 147.115 & 23.616 & 94.781 & 128.376 & CWG-WWG-da- 2.300 \\
\hline 0.0000 & -0.2937 & 0.2379 & 2.3000 & 2.5874 & 2.4759 & 23.278 & 125.284 & 144.503 & 28.036 & 96.439 & 123.976 & CWG-WWG-da- 2.300 \\
\hline 0.0833 & -0.2937 & 0.2379 & 2.3000 & 2.5874 & 2.4759 & 29.106 & 128.765 & 142.138 & 33.771 & 99.352 & 126.013 & CWG-WWG-da-2.300 \\
\hline 0.1667 & -0.2937 & 0.2379 & 2.3000 & 2.5874 & 2.4759 & 36.191 & 133.634 & 139.091 & 41.427 & 104.342 & 127.037 & CWG-WWG-da-2.300 \\
\hline 0.2500 & -0.2937 & 0.2379 & 2.3000 & 2.5874 & 2.4759 & 47.641 & 143.149 & 141.643 & 53.900 & 114.473 & 133.029 & CWG-WWG-da-2.300 \\
\hline-0.3500 & -0.2937 & 0.2379 & 2.5000 & 2.5874 & 2.4759 & 15.885 & 120.733 & 159.946 & 17.942 & 101.821 & 146.800 & CWG-WWG-da- 2.500 \\
\hline-0.2333 & -0.2937 & 0.2379 & 2.5000 & 2.5874 & 2.4759 & 14.094 & 116.924 & 151.234 & 13.786 & 95.379 & 134.834 & CWG-WWG-da-2.500 \\
\hline
\end{tabular}




\begin{tabular}{|c|c|c|c|c|c|c|c|c|c|c|c|c|}
\hline-0.1167 & -0.2937 & 0.2379 & 2.5000 & 2.5874 & 2.4759 & 17.756 & 123.394 & 149.525 & 21.297 & 99.306 & 131.144 & CWG-WWG-da- 2.500 \\
\hline 0.0000 & -0.2937 & 0.2379 & 2.5000 & 2.5874 & 2.4759 & 25.489 & 127.633 & 147.796 & 28.923 & 97.303 & 125.657 & CWG-WWG-da-2.500 \\
\hline 0.1167 & -0.2937 & 0.2379 & 2.5000 & 2.5874 & 2.4759 & 31.553 & 130.510 & 141.508 & 34.979 & 99.561 & 126.120 & CWG-WWG-da-2.500 \\
\hline 0.2333 & -0.2937 & 0.2379 & 2.5000 & 2.5874 & 2.4759 & 36.667 & 132.733 & 133.514 & 41.236 & 102.363 & 123.080 & CWG-WWG-da-2.500 \\
\hline 0.3500 & -0.2937 & 0.2379 & 2.5000 & 2.5874 & 2.4759 & 48.272 & 145.441 & 134.613 & 54.552 & 117.354 & 128.243 & CWG-WWG-da-2.500 \\
\hline-0.4274 & -0.2937 & 0.2379 & 2.6548 & 2.5874 & 2.4759 & 14.671 & 120.607 & 163.023 & 17.181 & 102.156 & 150.338 & CWG-WWG-da- 2.655 \\
\hline-0.2849 & -0.2937 & 0.2379 & 2.6548 & 2.5874 & 2.4759 & 14.706 & 118.498 & 155.692 & 14.248 & 96.776 & 139.072 & CWG-WWG-da- 2.655 \\
\hline-0.1425 & -0.2937 & 0.2379 & 2.6548 & 2.5874 & 2.4759 & 22.262 & 128.091 & 157.702 & 26.046 & 98.701 & 137.020 & CWG-WWG-da-2.655 \\
\hline 0.0000 & -0.2937 & 0.2379 & 2.6548 & 2.5874 & 2.4759 & 33.541 & 135.799 & 156.503 & 35.627 & 103.993 & 132.666 & CWG-WWG-da-2.655 \\
\hline 0.1425 & -0.2937 & 0.2379 & 2.6548 & 2.5874 & 2.4759 & 39.170 & 137.618 & 146.598 & 41.407 & 105.265 & 131.428 & CWG-WWG-da-2.655 \\
\hline 0.2849 & -0.2937 & 0.2379 & 2.6548 & 2.5874 & 2.4759 & 41.213 & 136.302 & 133.696 & 45.197 & 105.069 & 124.114 & CWG-WWG-da-2.655 \\
\hline 0.4274 & -0.2937 & 0.2379 & 2.6548 & 2.5874 & 2.4759 & 51.373 & 147.511 & 132.680 & 57.655 & 119.128 & 127.663 & CWG-WWG-da- 2.655 \\
\hline-0.3300 & 0.2937 & -0.2500 & 2.6600 & 2.5874 & 2.3000 & 29.784 & 136.366 & 174.866 & 32.900 & 107.049 & 154.431 & CSS-CSG-da-2.300 \\
\hline-0.3300 & 0.2937 & -0.1667 & 2.6600 & 2.5874 & 2.3000 & 21.341 & 127.680 & 167.929 & 23.438 & 97.277 & 146.311 & CSS-CSG-da-2.300 \\
\hline-0.3300 & 0.2937 & -0.0833 & 2.6600 & 2.5874 & 2.3000 & 17.871 & 125.955 & 166.152 & 19.346 & 95.886 & 143.707 & CSS-CSG-da- 2.300 \\
\hline-0.3300 & 0.2937 & 0.0000 & 2.6600 & 2.5874 & 2.3000 & 16.012 & 123.798 & 166.072 & 17.474 & 93.665 & 143.371 & CSS-CSG-da- 2.300 \\
\hline-0.3300 & 0.2937 & 0.0833 & 2.6600 & 2.5874 & 2.3000 & 15.779 & 120.193 & 166.532 & 16.340 & 89.603 & 145.634 & CSS-CSG-da- 2.300 \\
\hline-0.3300 & 0.2937 & 0.1667 & 2.6600 & 2.5874 & 2.3000 & 16.313 & 120.420 & 168.529 & 18.074 & 90.981 & 148.683 & CSS-CSG-da- 2.300 \\
\hline-0.3300 & 0.2937 & 0.2500 & 2.6600 & 2.5874 & 2.3000 & 21.031 & 125.930 & 175.677 & 25.908 & 97.955 & 155.754 & CSS-CSG-da-2.300 \\
\hline-0.3274 & 0.2937 & -0.3380 & 2.6548 & 2.5874 & 2.4759 & 28.040 & 134.707 & 171.242 & 31.164 & 109.733 & 150.935 & CSS-CSG-da- 2.476 \\
\hline-0.3274 & 0.2937 & -0.2253 & 2.6548 & 2.5874 & 2.4759 & 19.561 & 126.367 & 164.573 & 21.113 & 95.449 & 142.516 & CSS-CSG-da-2.476 \\
\hline-0.3274 & 0.2937 & -0.1127 & 2.6548 & 2.5874 & 2.4759 & 18.375 & 124.805 & 165.549 & 18.847 & 92.713 & 142.155 & CSS-CSG-da- 2.476 \\
\hline-0.3274 & 0.2937 & -0.0000 & 2.6548 & 2.5874 & 2.4759 & 17.253 & 125.298 & 166.779 & 17.613 & 94.042 & 142.948 & CSS-CSG-da- 2.476 \\
\hline-0.3274 & 0.2937 & 0.1127 & 2.6548 & 2.5874 & 2.4759 & 15.129 & 119.690 & 165.900 & 14.963 & 88.325 & 144.142 & CSS-CSG-da- 2.476 \\
\hline-0.3274 & 0.2937 & 0.2253 & 2.6548 & 2.5874 & 2.4759 & 11.400 & 116.596 & 165.108 & 14.637 & 86.993 & 143.570 & CSS-CSG-da- 2.476 \\
\hline-0.3274 & 0.2937 & 0.3380 & 2.6548 & 2.5874 & 2.4759 & 16.513 & 121.394 & 171.710 & 21.912 & 93.881 & 152.143 & CSS-CSG-da- 2.476 \\
\hline-0.3300 & 0.2937 & -0.4250 & 2.6600 & 2.5874 & 2.6500 & 34.516 & 137.232 & 171.935 & 30.583 & 112.347 & 151.723 & CSS-CSG-da-2.650 \\
\hline-0.3300 & 0.2937 & -0.2833 & 2.6600 & 2.5874 & 2.6500 & 27.942 & 130.021 & 167.066 & 21.353 & 102.856 & 144.480 & CSS-CSG-da- 2.650 \\
\hline-0.3300 & 0.2937 & -0.1417 & 2.6600 & 2.5874 & 2.6500 & 25.794 & 132.705 & 172.324 & 25.063 & 99.422 & 147.761 & CSS-CSG-da- 2.650 \\
\hline-0.3300 & 0.2937 & 0.0000 & 2.6600 & 2.5874 & 2.6500 & 26.015 & 132.376 & 175.438 & 24.981 & 98.672 & 150.164 & CSS-CSG-da- 2.650 \\
\hline-0.3300 & 0.2937 & 0.1417 & 2.6600 & 2.5874 & 2.6500 & 25.143 & 126.310 & 172.692 & 18.405 & 93.960 & 149.829 & CSS-CSG-da- 2.650 \\
\hline-0.3300 & 0.2937 & 0.2833 & 2.6600 & 2.5874 & 2.6500 & 13.124 & 118.472 & 167.511 & 16.123 & 88.599 & 145.590 & CSS-CSG-da-2.650 \\
\hline-0.3300 & 0.2937 & 0.4250 & 2.6600 & 2.5874 & 2.6500 & 20.868 & 121.133 & 172.131 & 19.463 & 93.993 & 152.824 & CSS-CSG-da- 2.650 \\
\hline-0.2500 & 0.2937 & -0.2379 & 2.3000 & 2.5874 & 2.4759 & 29.273 & 134.670 & 167.032 & 32.233 & 105.146 & 146.658 & CSS-WSS-da-2.300 \\
\hline-0.1667 & 0.2937 & -0.2379 & 2.3000 & 2.5874 & 2.4759 & 23.976 & 127.425 & 155.115 & 25.741 & 96.206 & 132.224 & CSS-WSS-da- 2.300 \\
\hline-0.0833 & 0.2937 & -0.2379 & 2.3000 & 2.5874 & 2.4759 & 23.981 & 125.217 & 147.420 & 24.903 & 92.612 & 123.046 & CSS-WSS-da- 2.300 \\
\hline 0.0000 & 0.2937 & -0.2379 & 2.3000 & 2.5874 & 2.4759 & 25.698 & 124.567 & 140.751 & 26.369 & 91.160 & 115.032 & CSS-WSS-da- 2.300 \\
\hline 0.0833 & 0.2937 & -0.2379 & 2.3000 & 2.5874 & 2.4759 & 27.840 & 124.369 & 131.987 & 28.968 & 90.898 & 115.854 & CSS-WSS-da- 2.300 \\
\hline 0.1667 & 0.2937 & -0.2379 & 2.3000 & 2.5874 & 2.4759 & 31.370 & 129.069 & 125.493 & 33.557 & 97.223 & 113.341 & CSS-WSS-da-2.300 \\
\hline 0.2500 & 0.2937 & -0.2379 & 2.3000 & 2.5874 & 2.4759 & 39.685 & 135.778 & 125.286 & 43.269 & 104.888 & 116.609 & CSS-WSS-da-2.300 \\
\hline-0.3500 & 0.2937 & -0.2380 & 2.5000 & 2.5874 & 2.4759 & 28.815 & 131.035 & 169.635 & 24.965 & 106.413 & 150.071 & CSS-WSS-da-2.500 \\
\hline-0.2333 & 0.2937 & -0.2380 & 2.5000 & 2.5874 & 2.4759 & 19.643 & 124.637 & 157.193 & 21.039 & 93.248 & 134.593 & CSS-WSS-da-2.500 \\
\hline-0.1167 & 0.2937 & -0.2380 & 2.5000 & 2.5874 & 2.4759 & 23.418 & 125.587 & 151.016 & 23.303 & 91.970 & 125.542 & CSS-WSS-da- 2.500 \\
\hline 0.0000 & 0.2937 & -0.2380 & 2.5000 & 2.5874 & 2.4759 & 27.285 & 126.273 & 143.482 & 26.642 & 91.396 & 116.072 & CSS-WSS-da-2.500 \\
\hline 0.1167 & 0.2937 & -0.2380 & 2.5000 & 2.5874 & 2.4759 & 28.376 & 127.355 & 129.692 & 28.512 & 93.711 & 113.959 & CSS-WSS-da-2.500 \\
\hline 0.2333 & 0.2937 & -0.2380 & 2.5000 & 2.5874 & 2.4759 & 28.872 & 125.442 & 117.648 & 30.786 & 92.965 & 106.902 & CSS-WSS-da-2.500 \\
\hline 0.3500 & 0.2937 & -0.2380 & 2.5000 & 2.5874 & 2.4759 & 36.761 & 132.369 & 115.879 & 40.802 & 99.156 & 109.277 & CSS-WSS-da-2.500 \\
\hline-0.4274 & 0.2937 & -0.2379 & 2.6548 & 2.5874 & 2.4759 & 28.385 & 131.845 & 174.376 & 24.807 & 107.527 & 155.138 & CSS-WSS-da- 2.655 \\
\hline-0.2849 & 0.2937 & -0.2379 & 2.6548 & 2.5874 & 2.4759 & 21.114 & 127.145 & 163.066 & 22.081 & 95.423 & 140.022 & CSS-WSS-da-2.655 \\
\hline-0.1425 & 0.2937 & -0.2379 & 2.6548 & 2.5874 & 2.4759 & 29.065 & 131.888 & 159.551 & 27.874 & 97.167 & 132.734 & CSS-WSS-da-2.655 \\
\hline 0.0000 & 0.2937 & -0.2379 & 2.6548 & 2.5874 & 2.4759 & 35.040 & 134.094 & 151.400 & 33.019 & 97.695 & 128.820 & CSS-WSS-da-2.655 \\
\hline 0.1425 & 0.2937 & -0.2379 & 2.6548 & 2.5874 & 2.4759 & 34.761 & 133.277 & 133.731 & 33.869 & 98.414 & 117.960 & CSS-WSS-da-2.655 \\
\hline 0.2849 & 0.2937 & -0.2379 & 2.6548 & 2.5874 & 2.4759 & 31.473 & 127.262 & 116.519 & 33.050 & 94.207 & 106.447 & CSS-WSS-da-2.655 \\
\hline 0.4274 & 0.2937 & -0.2379 & 2.6548 & 2.5874 & 2.4759 & 37.632 & 132.372 & 112.702 & 41.930 & 98.852 & 107.244 & CSS-WSS-da-2.655 \\
\hline-0.2500 & 0.2937 & 0.2379 & 2.3000 & 2.5874 & 2.4759 & 21.762 & 124.021 & 167.064 & 24.662 & 95.831 & 148.695 & CSG-WSG-da-2.300 \\
\hline-0.1667 & 0.2937 & 0.2379 & 2.3000 & 2.5874 & 2.4759 & 14.332 & 115.872 & 154.653 & 17.868 & 85.936 & 132.610 & CSG-WSG-da-2.300 \\
\hline-0.0833 & 0.2937 & 0.2379 & 2.3000 & 2.5874 & 2.4759 & 18.508 & 112.601 & 145.923 & 13.572 & 81.251 & 121.812 & CSG-WSG-da-2.300 \\
\hline 0.0000 & 0.2937 & 0.2379 & 2.3000 & 2.5874 & 2.4759 & 13.835 & 113.699 & 136.395 & 16.269 & 82.711 & 119.621 & CSG-WSG-da-2.300 \\
\hline 0.0833 & 0.2937 & 0.2379 & 2.3000 & 2.5874 & 2.4759 & 14.767 & 112.582 & 126.509 & 17.661 & 81.520 & 113.222 & CSG-WSG-da-2.300 \\
\hline 0.1667 & 0.2937 & 0.2379 & 2.3000 & 2.5874 & 2.4759 & 17.154 & 113.133 & 119.149 & 21.094 & 82.631 & 110.035 & CSG-WSG-da- 2.300 \\
\hline 0.2500 & 0.2937 & 0.2379 & 2.3000 & 2.5874 & 2.4759 & 24.465 & 119.924 & 118.241 & 29.777 & 88.031 & 112.479 & CSG-WSG-da-2.300 \\
\hline-0.3500 & 0.2937 & 0.2379 & 2.5000 & 2.5874 & 2.4759 & 15.983 & 121.559 & 170.582 & 21.090 & 94.118 & 151.526 & CSG-WSG-da-2.500 \\
\hline-0.2333 & 0.2937 & 0.2379 & 2.5000 & 2.5874 & 2.4759 & 10.445 & 113.643 & 157.158 & 13.595 & 83.572 & 135.079 & CSG-WSG-da-2.500 \\
\hline-0.1167 & 0.2937 & 0.2379 & 2.5000 & 2.5874 & 2.4759 & 12.938 & 113.261 & 149.667 & 14.557 & 80.896 & 124.750 & CSG-WSG-da-2.500 \\
\hline 0.0000 & 0.2937 & 0.2379 & 2.5000 & 2.5874 & 2.4759 & 15.268 & 112.410 & 138.779 & 16.371 & 78.794 & 121.052 & CSG-WSG-da-2.500 \\
\hline 0.1167 & 0.2937 & 0.2379 & 2.5000 & 2.5874 & 2.4759 & 14.758 & 112.042 & 123.802 & 16.658 & 79.754 & 111.184 & CSG-WSG-da-2.500 \\
\hline 0.2333 & 0.2937 & 0.2379 & 2.5000 & 2.5874 & 2.4759 & 13.811 & 108.791 & 110.761 & 17.477 & 77.688 & 102.940 & CSG-WSG-da-2.500 \\
\hline 0.3500 & 0.2937 & 0.2379 & 2.5000 & 2.5874 & 2.4759 & 20.537 & 114.782 & 108.255 & 26.299 & 82.542 & 104.445 & CSG-WSG-da-2.500 \\
\hline-0.4274 & 0.2937 & 0.2379 & 2.6548 & 2.5874 & 2.4759 & 15.984 & 124.775 & 175.558 & 21.275 & 98.780 & 156.834 & CSG-WSG-da-2.655 \\
\hline-0.2849 & 0.2937 & 0.2379 & 2.6548 & 2.5874 & 2.4759 & 13.348 & 116.526 & 162.996 & 14.379 & 86.140 & 142.050 & CSG-WSG-da-2.655 \\
\hline-0.1425 & 0.2937 & 0.2379 & 2.6548 & 2.5874 & 2.4759 & 18.746 & 119.771 & 158.250 & 19.265 & 86.288 & 131.955 & CSG-WSG-da-2.655 \\
\hline 0.0000 & 0.2937 & 0.2379 & 2.6548 & 2.5874 & 2.4759 & 22.933 & 120.174 & 146.638 & 22.645 & 85.023 & 120.278 & CSG-WSG-da- 2.655 \\
\hline 0.1425 & 0.2937 & 0.2379 & 2.6548 & 2.5874 & 2.4759 & 20.774 & 117.653 & 127.525 & 21.649 & 84.155 & 114.785 & CSG-WSG-da- 2.655 \\
\hline 0.2849 & 0.2937 & 0.2379 & 2.6548 & 2.5874 & 2.4759 & 15.855 & 110.146 & 109.277 & 19.185 & 78.486 & 102.031 & CSG-WSG-da-2.655 \\
\hline 0.4274 & 0.2937 & 0.2379 & 2.6548 & 2.5874 & 2.4759 & 20.783 & 114.237 & 104.733 & 26.797 & 81.657 & 101.976 & CSG-WSG-da- 2.655 \\
\hline 0.3274 & -0.2937 & -0.2500 & 2.6548 & 2.5874 & 2.3000 & 46.800 & 142.460 & 127.361 & 52.588 & 113.327 & 119.004 & WWS-WWG-da-2.300 \\
\hline 0.3274 & -0.2937 & -0.1667 & 2.6548 & 2.5874 & 2.3000 & 40.592 & 136.015 & 122.455 & 45.169 & 105.596 & 112.983 & WWS-WWG-da-2.300 \\
\hline 0.3274 & -0.2937 & -0.0833 & 2.6548 & 2.5874 & 2.3000 & 39.686 & 134.830 & 123.045 & 43.383 & 103.449 & 112.165 & WWS-WWG-da-2.300 \\
\hline 0.3274 & -0.2937 & -0.0000 & 2.6548 & 2.5874 & 2.3000 & 40.550 & 135.385 & 125.524 & 43.940 & 103.610 & 115.920 & WWS-WWG-da-2.300 \\
\hline 0.3274 & -0.2937 & 0.0833 & 2.6548 & 2.5874 & 2.3000 & 41.954 & 139.804 & 128.538 & 45.727 & 109.412 & 119.317 & WWS-WWG-da-2.300 \\
\hline 0.3274 & -0.2937 & 0.1667 & 2.6548 & 2.5874 & 2.3000 & 44.867 & 142.435 & 132.931 & 49.624 & 112.950 & 124.881 & WWS-WWG-da-2.300 \\
\hline 0.3274 & -0.2937 & 0.2500 & 2.6548 & 2.5874 & 2.3000 & 52.640 & 149.957 & 141.971 & 58.747 & 121.754 & 135.482 & WWS-WWG-da-2.300 \\
\hline 0.3274 & -0.2937 & -0.3380 & 2.6548 & 2.5874 & 2.4759 & 48.548 & 139.393 & 122.322 & 46.778 & 110.532 & 113.929 & WWS-WWG-da-2.476 \\
\hline 0.3274 & -0.2937 & -0.2253 & 2.6548 & 2.5874 & 2.4759 & 37.317 & 133.230 & 117.953 & 41.497 & 102.480 & 107.926 & WWS-WWG-da-2.476 \\
\hline
\end{tabular}




\begin{tabular}{|c|c|c|c|c|c|c|c|c|c|c|c|c|}
\hline 0.3274 & -0.2937 & -0.1127 & 2.6548 & 2.5874 & 2.4759 & 39.334 & 134.882 & 121.832 & 42.097 & 102.607 & 110.378 & WWS-WWG-da-2.476 \\
\hline 0.3274 & -0.2937 & -0.0000 & 2.6548 & 2.5874 & 2.4759 & 41.754 & 136.878 & 126.359 & 43.987 & 103.958 & 115.520 & WWS-WWG-da-2.476 \\
\hline 0.3274 & -0.2937 & 0.1127 & 2.6548 & 2.5874 & 2.4759 & 42.049 & 136.736 & 128.764 & 44.921 & 104.344 & 118.547 & WWS-WWG-da- 2.476 \\
\hline 0.3274 & -0.2937 & 0.2253 & 2.6548 & 2.5874 & 2.4759 & 42.255 & 139.907 & 130.824 & 46.698 & 110.071 & 122.448 & WWS-WWG-da- 2.476 \\
\hline 0.3274 & -0.2937 & 0.3380 & 2.6548 & 2.5874 & 2.4759 & 49.599 & 143.560 & 139.635 & 56.024 & 114.527 & 133.523 & WWS-WWG-da- 2.476 \\
\hline 0.3274 & -0.2937 & -0.4250 & 2.6548 & 2.5874 & 2.6500 & 43.650 & 143.635 & 121.573 & 49.761 & 116.006 & 108.692 & WWS-WWG-da-2.650 \\
\hline 0.3274 & -0.2937 & -0.2833 & 2.6548 & 2.5874 & 2.6500 & 44.865 & 135.970 & 119.131 & 40.846 & 104.779 & 108.416 & WWS-WWG-da-2.650 \\
\hline 0.3274 & -0.2937 & -0.1417 & 2.6548 & 2.5874 & 2.6500 & 45.954 & 141.920 & 127.679 & 47.539 & 108.502 & 114.972 & WWS-WWG-da-2.650 \\
\hline 0.3274 & -0.2937 & 0.0000 & 2.6548 & 2.5874 & 2.6500 & 50.445 & 145.867 & 134.731 & 51.175 & 111.454 & 122.249 & WWS-WWG-da-2.650 \\
\hline 0.3274 & -0.2937 & 0.1417 & 2.6548 & 2.5874 & 2.6500 & 49.036 & 143.895 & 135.904 & 50.740 & 110.322 & 124.415 & WWS-WWG-da- 2.650 \\
\hline 0.3274 & -0.2937 & 0.2833 & 2.6548 & 2.5874 & 2.6500 & 44.959 & 139.327 & 134.002 & 48.954 & 107.922 & 125.164 & WWS-WWG-da-2.650 \\
\hline 0.3274 & -0.2937 & 0.4250 & 2.6548 & 2.5874 & 2.6500 & 50.287 & 147.663 & 140.923 & 56.935 & 119.904 & 135.084 & WWS-WWG-da- 2.650 \\
\hline 0.3274 & -0.2500 & -0.2380 & 2.6548 & 2.3000 & 2.4759 & 45.257 & 140.584 & 125.331 & 50.480 & 110.797 & 116.147 & WWS-WSS-da-2.300 \\
\hline 0.3274 & -0.1667 & -0.2380 & 2.6548 & 2.3000 & 2.4759 & 37.154 & 131.867 & 117.423 & 40.879 & 100.430 & 108.182 & WWS-WSS-da-2.300 \\
\hline 0.3274 & -0.0833 & -0.2380 & 2.6548 & 2.3000 & 2.4759 & 34.158 & 131.548 & 114.608 & 36.680 & 99.805 & 104.273 & WWS-WSS-da-2.300 \\
\hline 0.3274 & 0.0000 & -0.2380 & 2.6548 & 2.3000 & 2.4759 & 32.905 & 129.624 & 113.496 & 34.754 & 97.054 & 102.956 & WWS-WSS-da-2.300 \\
\hline 0.3274 & 0.0833 & -0.2380 & 2.6548 & 2.3000 & 2.4759 & 32.360 & 128.429 & 113.037 & 34.186 & 95.689 & 102.959 & WWS-WSS-da-2.300 \\
\hline 0.3274 & 0.1667 & -0.2380 & 2.6548 & 2.3000 & 2.4759 & 33.664 & 129.148 & 114.371 & 36.050 & 96.836 & 105.288 & WWS-WSS-da-2.300 \\
\hline 0.3274 & 0.2500 & -0.2380 & 2.6548 & 2.3000 & 2.4759 & 40.253 & 135.246 & 120.950 & 43.588 & 103.768 & 113.171 & WWS-WSS-da-2.300 \\
\hline 0.3274 & -0.3250 & -0.2380 & 2.6548 & 2.4500 & 2.4759 & 43.417 & 139.413 & 123.617 & 49.206 & 110.324 & 114.993 & WWS-WSS-da-2.450 \\
\hline 0.3274 & -0.2167 & -0.2380 & 2.6548 & 2.4500 & 2.4759 & 35.183 & 130.458 & 115.653 & 38.981 & 99.197 & 104.755 & WWS-WSS-da-2.450 \\
\hline 0.3274 & -0.1083 & -0.2380 & 2.6548 & 2.4500 & 2.4759 & 34.108 & 128.522 & 114.860 & 36.226 & 95.387 & 104.076 & WWS-WSS-da- 2.450 \\
\hline 0.3274 & 0.0000 & -0.2380 & 2.6548 & 2.4500 & 2.4759 & 33.612 & 130.537 & 114.612 & 34.807 & 97.337 & 103.448 & WWS-WSS-da- 2.450 \\
\hline 0.3274 & 0.1083 & -0.2380 & 2.6548 & 2.4500 & 2.4759 & 31.745 & 127.844 & 112.914 & 33.017 & 94.542 & 102.421 & WWS-WSS-da- 2.450 \\
\hline 0.3274 & 0.2167 & -0.2380 & 2.6548 & 2.4500 & 2.4759 & 30.676 & 126.066 & 111.924 & 32.871 & 93.528 & 102.866 & WWS-WSS-da- 2.450 \\
\hline 0.3274 & 0.3250 & -0.2380 & 2.6548 & 2.4500 & 2.4759 & 43.077 & 128.321 & 118.363 & 38.381 & 96.001 & 111.080 & WWS-WSS-da-2.450 \\
\hline 0.3274 & -0.3937 & -0.2379 & 2.6548 & 2.5874 & 2.4759 & 49.661 & 140.887 & 124.571 & 48.193 & 112.361 & 116.374 & WWS-WSS-da-2.587 \\
\hline 0.3274 & -0.2625 & -0.2379 & 2.6548 & 2.5874 & 2.4759 & 37.049 & 132.788 & 117.576 & 40.841 & 101.609 & 107.220 & WWS-WSS-da-2.587 \\
\hline 0.3274 & -0.1312 & -0.2379 & 2.6548 & 2.5874 & 2.4759 & 38.803 & 133.542 & 119.691 & 40.427 & 99.963 & 108.471 & WWS-WSS-da-2.587 \\
\hline 0.3274 & 0.0000 & -0.2379 & 2.6548 & 2.5874 & 2.4759 & 39.393 & 133.019 & 120.637 & 39.833 & 98.031 & 108.719 & WWS-WSS-da-2.587 \\
\hline 0.3274 & 0.1312 & -0.2379 & 2.6548 & 2.5874 & 2.4759 & 35.913 & 132.016 & 117.428 & 36.576 & 98.094 & 106.417 & WWS-WSS-da-2.587 \\
\hline 0.3274 & 0.2625 & -0.2379 & 2.6548 & 2.5874 & 2.4759 & 37.521 & 123.330 & 113.299 & 31.316 & 89.518 & 104.189 & WWS-WSS-da-2.587 \\
\hline 0.3274 & 0.3937 & -0.2379 & 2.6548 & 2.5874 & 2.4759 & 36.917 & 132.539 & 118.641 & 40.724 & 99.040 & 111.755 & WWS-WSS-da-2.587 \\
\hline 0.3274 & -0.2500 & 0.2379 & 2.6548 & 2.3000 & 2.4759 & 49.481 & 146.532 & 138.335 & 55.274 & 117.952 & 131.430 & WWG-WSG-da- 2.300 \\
\hline 0.3274 & -0.1667 & 0.2379 & 2.6548 & 2.3000 & 2.4759 & 38.530 & 135.000 & 127.593 & 42.929 & 104.870 & 119.741 & WWG-WSG-da-2.300 \\
\hline 0.3274 & -0.0833 & 0.2379 & 2.6548 & 2.3000 & 2.4759 & 38.069 & 124.596 & 121.505 & 33.305 & 92.194 & 113.117 & WWG-WSG-da- 2.300 \\
\hline 0.3274 & 0.0000 & 0.2379 & 2.6548 & 2.3000 & 2.4759 & 27.454 & 122.626 & 116.880 & 30.376 & 90.715 & 108.559 & WWG-WSG-da- 2.300 \\
\hline 0.3274 & 0.0833 & 0.2379 & 2.6548 & 2.3000 & 2.4759 & 23.404 & 118.968 & 112.944 & 26.549 & 84.770 & 105.329 & WWG-WSG-da-2.300 \\
\hline 0.3274 & 0.1667 & 0.2379 & 2.6548 & 2.3000 & 2.4759 & 21.491 & 116.525 & 111.096 & 25.412 & 82.852 & 104.675 & WWG-WSG-da- 2.300 \\
\hline 0.3274 & 0.2500 & 0.2379 & 2.6548 & 2.3000 & 2.4759 & 25.320 & 119.916 & 114.956 & 30.350 & 87.135 & 109.980 & WWG-WSG-da-2.300 \\
\hline 0.3274 & -0.3000 & 0.2380 & 2.6548 & 2.4000 & 2.4759 & 49.141 & 146.618 & 137.905 & 55.214 & 118.410 & 131.085 & WWG-WSG-da-2.400 \\
\hline 0.3274 & -0.2000 & 0.2380 & 2.6548 & 2.4000 & 2.4759 & 37.674 & 134.491 & 126.714 & 42.014 & 104.372 & 118.655 & WWG-WSG-da- 2.400 \\
\hline 0.3274 & -0.1000 & 0.2380 & 2.6548 & 2.4000 & 2.4759 & 37.799 & 124.611 & 121.341 & 32.728 & 91.897 & 112.523 & WWG-WSG-da- 2.400 \\
\hline 0.3274 & 0.0000 & 0.2380 & 2.6548 & 2.4000 & 2.4759 & 27.054 & 122.334 & 116.614 & 29.514 & 89.982 & 107.838 & WWG-WSG-da- 2.400 \\
\hline 0.3274 & 0.1000 & 0.2380 & 2.6548 & 2.4000 & 2.4759 & 21.577 & 117.147 & 111.309 & 24.389 & 82.590 & 103.437 & WWG-WSG-da- 2.400 \\
\hline 0.3274 & 0.2000 & 0.2380 & 2.6548 & 2.4000 & 2.4759 & 17.782 & 112.753 & 107.614 & 21.648 & 78.967 & 101.258 & WWG-WSG-da- 2.400 \\
\hline 0.3274 & 0.3000 & 0.2380 & 2.6548 & 2.4000 & 2.4759 & 21.383 & 115.880 & 111.265 & 26.671 & 83.281 & 106.680 & WWG-WSG-da-2.400 \\
\hline 0.3274 & -0.3937 & 0.2379 & 2.6548 & 2.5874 & 2.4759 & 52.051 & 146.933 & 140.520 & 58.551 & 118.220 & 133.843 & WWG-WSG-da-2.587 \\
\hline 0.3274 & -0.2625 & 0.2379 & 2.6548 & 2.5874 & 2.4759 & 41.254 & 138.663 & 130.108 & 45.346 & 108.409 & 121.557 & WWG-WSG-da-2.587 \\
\hline 0.3274 & -0.1312 & 0.2379 & 2.6548 & 2.5874 & 2.4759 & 38.411 & 131.396 & 127.708 & 40.515 & 97.833 & 117.849 & WWG-WSG-da- 2.587 \\
\hline 0.3274 & 0.0000 & 0.2379 & 2.6548 & 2.5874 & 2.4759 & 33.731 & 129.171 & 123.442 & 35.039 & 95.683 & 113.515 & WWG-WSG-da- 2.587 \\
\hline 0.3274 & 0.1312 & 0.2379 & 2.6548 & 2.5874 & 2.4759 & 25.109 & 119.641 & 115.126 & 27.097 & 86.659 & 106.543 & WWG-WSG-da- 2.587 \\
\hline 0.3274 & 0.2625 & 0.2379 & 2.6548 & 2.5874 & 2.4759 & 16.488 & 111.338 & 106.687 & 20.157 & 77.250 & 100.308 & WWG-WSG-da-2.587 \\
\hline 0.3274 & 0.3937 & 0.2379 & 2.6548 & 2.5874 & 2.4759 & 18.453 & 112.791 & 108.748 & 24.166 & 80.493 & 104.776 & WWG-WSG-da- 2.587 \\
\hline 0.3274 & 0.2937 & -0.2500 & 2.6548 & 2.5874 & 2.3000 & 41.168 & 136.043 & 122.855 & 44.972 & 105.072 & 115.715 & WSS-WSG-da-2.300 \\
\hline 0.3274 & 0.2937 & -0.1667 & 2.6548 & 2.5874 & 2.3000 & 31.501 & 126.159 & 114.635 & 34.268 & 94.084 & 106.634 & WSS-WSG-da-2.300 \\
\hline 0.3274 & 0.2937 & -0.0833 & 2.6548 & 2.5874 & 2.3000 & 26.648 & 121.055 & 111.396 & 28.786 & 88.283 & 102.952 & WSS-WSG-da- 2.300 \\
\hline 0.3274 & 0.2937 & -0.0000 & 2.6548 & 2.5874 & 2.3000 & 23.326 & 117.467 & 109.757 & 25.454 & 84.620 & 101.492 & WSS-WSG-da-2.300 \\
\hline 0.3274 & 0.2937 & 0.0833 & 2.6548 & 2.5874 & 2.3000 & 20.639 & 115.541 & 108.692 & 23.433 & 80.706 & 101.288 & WSS-WSG-da-2.300 \\
\hline 0.3274 & 0.2937 & 0.1667 & 2.6548 & 2.5874 & 2.3000 & 19.863 & 114.542 & 109.363 & 23.859 & 80.784 & 103.360 & WSS-WSG-da-2.300 \\
\hline 0.3274 & 0.2937 & 0.2500 & 2.6548 & 2.5874 & 2.3000 & 24.530 & 119.015 & 115.243 & 30.025 & 86.645 & 110.927 & WSS-WSG-da-2.300 \\
\hline 0.3274 & 0.2937 & -0.3380 & 2.6548 & 2.5874 & 2.4759 & 40.805 & 137.074 & 120.961 & 44.640 & 103.927 & 113.616 & WSS-WSG-da-2.476 \\
\hline 0.3274 & 0.2937 & -0.2253 & 2.6548 & 2.5874 & 2.4759 & 36.785 & 122.358 & 112.770 & 30.803 & 88.745 & 104.044 & WSS-WSG-da-2.476 \\
\hline 0.3274 & 0.2937 & -0.1127 & 2.6548 & 2.5874 & 2.4759 & 27.924 & 119.054 & 111.891 & 29.076 & 84.264 & 102.316 & WSS-WSG-da-2.476 \\
\hline 0.3274 & 0.2937 & -0.0000 & 2.6548 & 2.5874 & 2.4759 & 24.880 & 119.271 & 111.064 & 25.936 & 85.337 & 101.633 & WSS-WSG-da-2.476 \\
\hline 0.3274 & 0.2937 & 0.1127 & 2.6548 & 2.5874 & 2.4759 & 19.888 & 114.921 & 108.172 & 21.975 & 79.356 & 100.027 & WSS-WSG-da-2.476 \\
\hline 0.3274 & 0.2937 & 0.2253 & 2.6548 & 2.5874 & 2.4759 & 15.585 & 110.327 & 105.640 & 19.496 & 76.429 & 99.574 & WSS-WSG-da-2.476 \\
\hline 0.3274 & 0.2937 & 0.3380 & 2.6548 & 2.5874 & 2.4759 & 20.555 & 113.713 & 102.413 & 25.160 & 82.938 & 105.574 & WSS-WSG-da-2.476 \\
\hline 0.3274 & 0.2937 & -0.4250 & 2.6548 & 2.5874 & 2.6500 & 43.876 & 140.594 & 122.718 & 47.740 & 107.593 & 115.191 & WSS-WSG-da-2.650 \\
\hline 0.3274 & 0.2937 & -0.2833 & 2.6548 & 2.5874 & 2.6500 & 35.331 & 131.757 & 116.152 & 36.978 & 96.368 & 106.656 & WSS-WSG-da-2.650 \\
\hline 0.3274 & 0.2937 & -0.1417 & 2.6548 & 2.5874 & 2.6500 & 35.918 & 131.957 & 119.211 & 35.875 & 94.664 & 108.311 & WSS-WSG-da-2.650 \\
\hline 0.3274 & 0.2937 & 0.0000 & 2.6548 & 2.5874 & 2.6500 & 33.776 & 129.370 & 119.792 & 33.461 & 91.579 & 108.906 & WSS-WSG-da-2.650 \\
\hline 0.3274 & 0.2937 & 0.1417 & 2.6548 & 2.5874 & 2.6500 & 26.011 & 121.178 & 114.560 & 27.170 & 84.652 & 105.454 & WSS-WSG-da-2.650 \\
\hline 0.3274 & 0.2937 & 0.2833 & 2.6548 & 2.5874 & 2.6500 & 16.816 & 111.629 & 107.380 & 20.508 & 77.462 & 101.120 & WSS-WSG-da-2.650 \\
\hline 0.3274 & 0.2937 & 0.4250 & 2.6548 & 2.5874 & 2.6500 & 18.508 & 113.048 & 102.085 & 24.958 & 81.501 & 105.680 & WSS-WSG-da-2.650 \\
\hline
\end{tabular}




\section{Protonation states; energies and geometries}

Absolute energies are given in hartrees. CASSCF and CASPT2 calculations correspond to the $(6,6)$ active space. Geometries are in $\AA$.

\section{Protonation state: CWS}

$\begin{array}{ll}\text { CASSCF } & \\ \mathrm{S}_{0} & -1059.86552445 \\ { }^{1} \pi \pi^{*} & -1059.69664819 \\ { }^{1} \pi \sigma^{*} & -1059.65051450\end{array}$

$\begin{array}{cc}\text { CASPT2 } & \\ \mathrm{S}_{0} & -1061.8908613827 \\ { }^{1} \pi \pi^{*} & -1061.7553308705 \\ { }^{1} \pi \sigma^{*} & -1061.6998849058\end{array}$

0.000000

0.000000

0.000000

0.000000

0.000000

0.000000

0.000000

0.000000

0.000000

0.000000

0.000000

0.000000

0.000000

0.000000

0.000000

0.000000

0.000000

0.000000

0.000000

0.890577

0.000000

0.000000

$-0.890577$

0.883744

0.000000

0.000000

$-0.883744$

0.000000

0.000000

0.000000

0.000000

0.000000

0.000000

0.000000

0.000000

0.000000

0.000000

0.000000 


\section{Protonation state: $\mathrm{CWG}$}

\section{CASSCF}

$\begin{array}{ll}\mathrm{S}_{0} & -1059.84523695 \\ { }^{1} \pi \pi^{*} & -1059.67900154 \\ { }^{1} \pi \sigma^{*} & -1059.61619091\end{array}$

\section{CASPT2 \\ $\begin{array}{ll}\mathrm{S}_{0} & -1061.8709745851\end{array}$ \\ ${ }^{1} \pi \pi^{*} \quad-1061.7384883306$ \\ ${ }^{1} \pi \sigma^{*} \quad-1061.6672777496$}

$\begin{array}{lrrr}\mathrm{C} & 3.468090 & -2.549320 & 0.000000 \\ \mathrm{~N} & 2.726662 & -1.491082 & 0.000000 \\ \mathrm{~N} & 4.832023 & -2.287040 & 0.000000 \\ \mathrm{C} & 5.015243 & -0.893059 & 0.000000 \\ \mathrm{O} & 6.093525 & -0.309937 & 0.000000 \\ \mathrm{C} & 3.613793 & -0.402423 & 0.000000 \\ \mathrm{C} & 3.292751 & 0.922997 & 0.000000 \\ \mathrm{H} & 4.159717 & 1.583850 & 0.000000 \\ \mathrm{C} & 2.004716 & 1.569785 & 0.000000 \\ \mathrm{C} & 0.766429 & 0.877720 & 0.000000 \\ \mathrm{H} & 0.760228 & -0.206555 & 0.000000 \\ \mathrm{C} & 1.966439 & 2.984347 & 0.000000 \\ \mathrm{H} & 2.904090 & 3.536425 & 0.000000 \\ \mathrm{C} & -0.438217 & 1.563377 & 0.000000 \\ \mathrm{H} & -1.367575 & 1.000328 & 0.000000 \\ \mathrm{C} & 0.768943 & 3.676846 & 0.000000 \\ \mathrm{H} & 0.741936 & 4.762169 & 0.000000 \\ \mathrm{C} & -0.450232 & 2.973064 & 0.000000 \\ \mathrm{H} & 5.585231 & -2.956244 & 0.000000 \\ \mathrm{H} & -6.464509 & -0.217188 & 0.890577 \\ \mathrm{C} & -5.911319 & -0.554915 & 0.000000 \\ \mathrm{H} & -5.921203 & -1.651819 & 0.000000 \\ \mathrm{H} & -6.464509 & -0.217188 & -0.890577 \\ \mathrm{H} & -4.496134 & -3.453914 & 0.883744 \\ \mathrm{C} & -3.901074 & -3.712850 & 0.000000 \\ \mathrm{H} & -3.709543 & -4.788010 & 0.000000 \\ \mathrm{H} & -4.496134 & -3.453914 & -0.883744 \\ \mathrm{C} & -2.576422 & -2.918985 & 0.000000 \\ \mathrm{H} & 3.085545 & -3.563982 & 0.000000 \\ \mathrm{O} & -1.583595 & 3.703313 & 0.000000 \\ \mathrm{O} & -3.918497 & 2.439907 & 0.000000 \\ \mathrm{O} & -4.595721 & -0.057293 & 0.000000 \\ \mathrm{O} & -2.689540 & -1.637342 & 0.000000 \\ \mathrm{O} & -1.509676 & -3.558546 & 0.000000 \\ \mathrm{H} & -2.463097 & 3.227417 & 0.000000 \\ & -4.188411 & 1.444623 & 0.000000 \\ & -3.459434 & -0.999170 & 0.000000 \\ \mathrm{H} & -722907 & 2.967445 & 0.000000\end{array}$


Protonation state: CSS

\section{CASSCF}

$\begin{array}{ll}\mathrm{S}_{0} & -1059.82099626 \\ { }^{1} \pi \pi^{*} & -1059.65832290 \\ { }^{1} \pi \sigma^{*} & -1059.59928837\end{array}$

$\begin{array}{cc}\text { CASPT2 } & \\ \mathrm{S}_{0} & -1061.8568327578 \\ { }^{1} \pi \pi^{*} & -1061.7269503400 \\ { }^{1} \pi \sigma^{*} & -1061.6606174347\end{array}$

\begin{tabular}{lrrr}
$\mathrm{C}$ & 3.403357 & -2.492530 & 0.000000 \\
$\mathrm{~N}$ & 2.661929 & -1.434292 & 0.000000 \\
$\mathrm{~N}$ & 4.767290 & -2.230250 & 0.000000 \\
$\mathrm{C}$ & 4.950510 & -0.836269 & 0.000000 \\
$\mathrm{O}$ & 6.028792 & -0.253147 & 0.000000 \\
$\mathrm{C}$ & 3.549060 & -0.345633 & 0.000000 \\
$\mathrm{C}$ & 3.228018 & 0.979787 & 0.000000 \\
$\mathrm{H}$ & 4.094984 & 1.640640 & 0.000000 \\
$\mathrm{C}$ & 1.939983 & 1.626575 & 0.000000 \\
$\mathrm{C}$ & 0.701696 & 0.934510 & 0.000000 \\
$\mathrm{H}$ & 0.695495 & -0.149765 & 0.000000 \\
$\mathrm{C}$ & 1.901706 & 3.041137 & 0.000000 \\
$\mathrm{H}$ & 2.839357 & 3.593215 & 0.000000 \\
$\mathrm{C}$ & -0.502950 & 1.620167 & 0.000000 \\
$\mathrm{H}$ & -1.432308 & 1.057118 & 0.000000 \\
$\mathrm{C}$ & 0.704210 & 3.733636 & 0.000000 \\
$\mathrm{H}$ & 0.677203 & 4.818959 & 0.000000 \\
$\mathrm{C}$ & -0.514965 & 3.029854 & 0.000000 \\
$\mathrm{H}$ & 5.520498 & -2.899454 & 0.000000 \\
$\mathrm{H}$ & -6.533815 & -0.162872 & 0.890577 \\
$\mathrm{C}$ & -5.980625 & -0.500599 & 0.000000 \\
$\mathrm{H}$ & -5.990509 & -1.597503 & 0.000000 \\
$\mathrm{H}$ & -6.533815 & -0.162872 & -0.890577 \\
$\mathrm{H}$ & -4.431401 & -3.510704 & 0.883744 \\
$\mathrm{C}$ & -3.836341 & -3.769640 & 0.000000 \\
$\mathrm{H}$ & -3.644810 & -4.844800 & 0.000000 \\
$\mathrm{H}$ & -4.431401 & -3.510704 & -0.883744 \\
$\mathrm{C}$ & -2.511689 & -2.975775 & 0.000000 \\
$\mathrm{H}$ & 3.020812 & -3.507192 & 0.000000 \\
$\mathrm{O}$ & -1.648328 & 3.760103 & 0.000000 \\
$\mathrm{O}$ & -3.987803 & 2.494222 & 0.000000 \\
$\mathrm{O}$ & -4.665027 & -0.002977 & 0.000000 \\
$\mathrm{O}$ & -2.624807 & -1.694132 & 0.000000 \\
& -1.444943 & -3.615336 & 0.000000 \\
$\mathrm{H}$ & -2.527830 & 3.284208 & 0.000000 \\
$\mathrm{H}$ & -3.793288 & 0.962161 & 0.000000 \\
\hline
\end{tabular}




\section{Protonation state: WWS}

\section{CASSCF}

$\begin{array}{ll}\mathrm{S}_{0} & -1059.79402744 \\ { }^{1} \pi \pi^{*} & -1059.64884246 \\ { }^{1} \pi \sigma^{*} & -1059.67567730\end{array}$

\section{CASPT2 \\ $\mathrm{S}_{0} \quad-1061.8257687425$ \\ ${ }^{1} \pi \pi^{*} \quad-1061.7238847522$ \\ ${ }^{1} \pi \sigma^{*} \quad-1061.7180893118$}

\begin{tabular}{|c|c|c|c|}
\hline $\mathrm{C}$ & 3.401071 & -2.493767 & 0.000000 \\
\hline $\mathrm{N}$ & 2.659643 & -1.435529 & 0.000000 \\
\hline $\mathrm{N}$ & 4.765004 & -2.231487 & 0.000000 \\
\hline $\mathrm{C}$ & 4.948224 & -0.837506 & 0.000000 \\
\hline $\mathrm{O}$ & 6.026506 & -0.254384 & 0.000000 \\
\hline $\mathrm{C}$ & 3.546774 & -0.346870 & 0.000000 \\
\hline $\mathrm{C}$ & 3.225732 & 0.978550 & 0.000000 \\
\hline $\mathrm{H}$ & 4.092698 & 1.639403 & 0.000000 \\
\hline $\mathrm{C}$ & 1.937697 & 1.625338 & 0.000000 \\
\hline $\mathrm{C}$ & 0.699410 & 0.933273 & 0.000000 \\
\hline $\mathrm{H}$ & 0.693209 & -0.151002 & 0.000000 \\
\hline $\mathrm{C}$ & 1.899420 & 3.039900 & 0.000000 \\
\hline $\mathrm{H}$ & 2.837071 & 3.591978 & 0.000000 \\
\hline $\mathrm{C}$ & -0.505236 & 1.618930 & 0.000000 \\
\hline $\mathrm{H}$ & -1.434594 & 1.055881 & 0.000000 \\
\hline $\mathrm{C}$ & 0.701924 & 3.732399 & 0.000000 \\
\hline $\mathrm{H}$ & 0.674917 & 4.817722 & 0.000000 \\
\hline $\mathrm{C}$ & -0.517251 & 3.028617 & 0.000000 \\
\hline $\mathrm{H}$ & 5.518212 & -2.900691 & 0.000000 \\
\hline $\mathrm{H}$ & -6.531528 & -0.161635 & 0.890577 \\
\hline $\mathrm{C}$ & -5.978338 & -0.499362 & 0.000000 \\
\hline $\mathrm{H}$ & -5.988222 & -1.596266 & 0.000000 \\
\hline $\mathrm{H}$ & -6.531528 & -0.161635 & -0.890577 \\
\hline $\mathrm{H}$ & -4.429115 & -3.509467 & 0.883744 \\
\hline $\mathrm{C}$ & -3.834055 & -3.768403 & 0.000000 \\
\hline $\mathrm{H}$ & -3.642524 & -4.843563 & 0.000000 \\
\hline $\mathrm{H}$ & -4.429115 & -3.509467 & -0.883744 \\
\hline $\mathrm{C}$ & -2.509403 & -2.974538 & 0.000000 \\
\hline $\mathrm{H}$ & 3.018526 & -3.508429 & 0.000000 \\
\hline $\mathrm{O}$ & -1.650614 & 3.758866 & 0.000000 \\
\hline $\mathrm{O}$ & -3.985516 & 2.495460 & 0.000000 \\
\hline $\mathrm{O}$ & -4.662740 & -0.001740 & 0.000000 \\
\hline $\mathrm{O}$ & -2.622521 & -1.692895 & 0.000000 \\
\hline $\mathrm{O}$ & -1.442657 & -3.614099 & 0.000000 \\
\hline $\mathrm{H}$ & -3.106014 & 2.971355 & 0.000000 \\
\hline $\mathrm{H}$ & -4.247256 & 1.530321 & 0.000000 \\
\hline $\mathrm{H}$ & -3.860767 & -0.666502 & 0.000000 \\
\hline $\mathrm{H}$ & -4.789926 & 3.022998 & 0.000000 \\
\hline
\end{tabular}




\section{Protonation state: CSG}

\section{CASSCF}

$\begin{array}{ll}\mathrm{S}_{0} & -1059.84425268 \\ { }^{1} \pi \pi^{*} & -1059.67982799 \\ { }^{1} \pi \sigma^{*} & -1059.60577349\end{array}$

$\begin{array}{cc}\text { CASPT2 } & \\ \mathrm{S}_{0} & -1061.8679461930 \\ { }^{1} \pi \pi^{*} & -1061.7535890055 \\ { }^{1} \pi \sigma^{*} & -1061.6644908982\end{array}$

\begin{tabular}{lrrr}
$\mathrm{C}$ & 3.468090 & -2.549320 & 0.000000 \\
$\mathrm{~N}$ & 2.726662 & -1.491082 & 0.000000 \\
$\mathrm{~N}$ & 4.832023 & -2.287040 & 0.000000 \\
$\mathrm{C}$ & 5.015243 & -0.893059 & 0.000000 \\
$\mathrm{O}$ & 6.093525 & -0.309937 & 0.000000 \\
$\mathrm{C}$ & 3.613793 & -0.402423 & 0.000000 \\
$\mathrm{C}$ & 3.292751 & 0.922997 & 0.000000 \\
$\mathrm{H}$ & 4.159717 & 1.583850 & 0.000000 \\
$\mathrm{C}$ & 2.004716 & 1.569785 & 0.000000 \\
$\mathrm{C}$ & 0.766429 & 0.877720 & 0.000000 \\
$\mathrm{H}$ & 0.760228 & -0.206555 & 0.000000 \\
$\mathrm{C}$ & 1.966439 & 2.984347 & 0.000000 \\
$\mathrm{H}$ & 2.904090 & 3.536425 & 0.000000 \\
$\mathrm{C}$ & -0.438217 & 1.563377 & 0.000000 \\
$\mathrm{H}$ & -1.367575 & 1.000328 & 0.000000 \\
$\mathrm{C}$ & 0.768943 & 3.676846 & 0.000000 \\
$\mathrm{H}$ & 0.741936 & 4.762169 & 0.000000 \\
$\mathrm{C}$ & -0.450232 & 2.973064 & 0.000000 \\
$\mathrm{H}$ & 5.585231 & -2.956244 & 0.000000 \\
$\mathrm{H}$ & -6.464509 & -0.217188 & 0.890577 \\
$\mathrm{C}$ & -5.911319 & -0.554915 & 0.000000 \\
$\mathrm{H}$ & -5.921203 & -1.651819 & 0.000000 \\
$\mathrm{H}$ & -6.464509 & -0.217188 & -0.890577 \\
$\mathrm{H}$ & -4.496134 & -3.453914 & 0.883744 \\
$\mathrm{C}$ & -3.901074 & -3.712850 & 0.000000 \\
$\mathrm{H}$ & -3.709543 & -4.788010 & 0.000000 \\
$\mathrm{H}$ & -4.496134 & -3.453914 & -0.883744 \\
$\mathrm{C}$ & -2.576422 & -2.918985 & 0.000000 \\
$\mathrm{H}$ & 3.085545 & -3.563982 & 0.000000 \\
$\mathrm{O}$ & -1.583595 & 3.703313 & 0.000000 \\
$\mathrm{O}$ & -3.918497 & 2.439907 & 0.000000 \\
$\mathrm{O}$ & -4.595721 & -0.057293 & 0.000000 \\
$\mathrm{O}$ & -2.689540 & -1.637342 & 0.000000 \\
& -1.509676 & -3.558546 & 0.000000 \\
$\mathrm{H}$ & -2.500446 & 3.207208 & 0.000000 \\
$\mathrm{H}$ & -3.459434 & -0.999170 & 0.000000 \\
& & & 0.00000000 \\
\hline
\end{tabular}




\section{Protonation state: WWG}

\section{CASSCF}

$\begin{array}{ll}\mathrm{S}_{0} & -1059.79978152 \\ { }^{1} \pi \pi^{*} & -1059.64455009 \\ { }^{1} \pi \sigma^{*} & -1059.65818429\end{array}$

\section{CASPT2 \\ $\mathrm{S}_{0} \quad-1061.8185982115$ \\ ${ }^{1} \pi \pi^{*} \quad-1061.7181007686$ \\ ${ }^{1} \pi \sigma^{*} \quad-1061.6971472828$}

$\begin{array}{lrrr}\mathrm{C} & 3.468090 & -2.549320 & 0.000000 \\ \mathrm{~N} & 2.726662 & -1.491082 & 0.000000 \\ \mathrm{~N} & 4.832023 & -2.287040 & 0.000000 \\ \mathrm{C} & 5.015243 & -0.893059 & 0.000000 \\ \mathrm{O} & 6.093525 & -0.309937 & 0.000000 \\ \mathrm{C} & 3.613793 & -0.402423 & 0.000000 \\ \mathrm{C} & 3.292751 & 0.922997 & 0.000000 \\ \mathrm{H} & 4.159717 & 1.583850 & 0.000000 \\ \mathrm{C} & 2.004716 & 1.569785 & 0.000000 \\ \mathrm{C} & 0.766429 & 0.877720 & 0.000000 \\ \mathrm{H} & 0.760228 & -0.206555 & 0.000000 \\ \mathrm{C} & 1.966439 & 2.984347 & 0.000000 \\ \mathrm{H} & 2.904090 & 3.536425 & 0.000000 \\ \mathrm{C} & -0.438217 & 1.563377 & 0.000000 \\ \mathrm{H} & -1.367575 & 1.000328 & 0.000000 \\ \mathrm{C} & 0.768943 & 3.676846 & 0.000000 \\ \mathrm{H} & 0.741936 & 4.762169 & 0.000000 \\ \mathrm{C} & -0.450232 & 2.973064 & 0.000000 \\ \mathrm{H} & 5.585231 & -2.956244 & 0.000000 \\ \mathrm{H} & -6.464509 & -0.217188 & 0.890577 \\ \mathrm{C} & -5.911319 & -0.554915 & 0.000000 \\ \mathrm{H} & -5.921203 & -1.651819 & 0.000000 \\ \mathrm{H} & -6.464509 & -0.217188 & -0.890577 \\ \mathrm{H} & -4.496134 & -3.453914 & 0.883744 \\ \mathrm{C} & -3.901074 & -3.712850 & 0.000000 \\ \mathrm{H} & -3.709543 & -4.788010 & 0.000000 \\ \mathrm{H} & -4.496134 & -3.453914 & -0.883744 \\ \mathrm{C} & -2.576422 & -2.918985 & 0.000000 \\ \mathrm{H} & 3.085545 & -3.563982 & 0.000000 \\ \mathrm{O} & -1.583595 & 3.703313 & 0.000000 \\ \mathrm{O} & -3.918497 & 2.439907 & 0.000000 \\ \mathrm{O} & -4.595721 & -0.057293 & 0.000000 \\ \mathrm{O} & -2.689540 & -1.637342 & 0.000000 \\ \mathrm{H} & -1.509676 & -3.558546 & 0.000000 \\ & -4.188411 & 1.444623 & 0.000000 \\ \mathrm{H} & -3.459434 & -0.999170 & 0.000000 \\ & & & 0.000000\end{array}$




\section{Protonation state: WSS}

\section{CASSCF}

$\begin{array}{ll}\mathrm{S}_{0} & -1059.80922139 \\ { }^{1} \pi \pi^{*} & -1059.65555569 \\ { }^{1} \pi \sigma^{*} & -1059.68042361\end{array}$

$\begin{array}{cc}\text { CASPT2 } & \\ \mathrm{S}_{0} & -1061.8319326148 \\ { }^{1} \pi \pi^{*} & -1061.7372896975 \\ { }^{1} \pi \sigma^{*} & -1061.7208937232\end{array}$

$\begin{array}{lrrr}\mathrm{C} & 3.401071 & -2.493767 & 0.000000 \\ \mathrm{~N} & 2.659643 & -1.435529 & 0.000000 \\ \mathrm{~N} & 4.765004 & -2.231487 & 0.000000 \\ \mathrm{C} & 4.948224 & -0.837506 & 0.000000 \\ \mathrm{O} & 6.026506 & -0.254384 & 0.000000 \\ \mathrm{C} & 3.546774 & -0.346870 & 0.000000 \\ \mathrm{C} & 3.225732 & 0.978550 & 0.000000 \\ \mathrm{H} & 4.092698 & 1.639403 & 0.000000 \\ \mathrm{C} & 1.937697 & 1.625338 & 0.000000 \\ \mathrm{C} & 0.699410 & 0.933273 & 0.000000 \\ \mathrm{H} & 0.693209 & -0.151002 & 0.000000 \\ \mathrm{C} & 1.899420 & 3.039900 & 0.000000 \\ \mathrm{H} & 2.837071 & 3.591978 & 0.000000 \\ \mathrm{C} & -0.505236 & 1.618930 & 0.000000 \\ \mathrm{H} & -1.434594 & 1.055881 & 0.000000 \\ \mathrm{C} & 0.701924 & 3.732399 & 0.000000 \\ \mathrm{H} & 0.674917 & 4.817722 & 0.000000 \\ \mathrm{C} & -0.517251 & 3.028617 & 0.000000 \\ \mathrm{H} & 5.518212 & -2.900691 & 0.000000 \\ \mathrm{H} & -6.531528 & -0.161635 & 0.890577 \\ \mathrm{C} & -5.978338 & -0.499362 & 0.000000 \\ \mathrm{H} & -5.988222 & -1.596266 & 0.000000 \\ \mathrm{H} & -6.531528 & -0.161635 & -0.890577 \\ \mathrm{H} & -4.429115 & -3.509467 & 0.883744 \\ \mathrm{C} & -3.834055 & -3.768403 & 0.000000 \\ \mathrm{H} & -3.642524 & -4.843563 & 0.000000 \\ \mathrm{H} & -4.429115 & -3.509467 & -0.883744 \\ \mathrm{C} & -2.509403 & -2.974538 & 0.000000 \\ \mathrm{H} & 3.018526 & -3.508429 & 0.000000 \\ \mathrm{O} & -1.650614 & 3.758866 & 0.000000 \\ \mathrm{O} & -3.985516 & 2.495460 & 0.000000 \\ \mathrm{O} & -4.662740 & -0.001740 & 0.000000 \\ \mathrm{O} & -2.622521 & -1.692895 & 0.000000 \\ & -1.442657 & -3.614099 & 0.000000 \\ \mathrm{H} & -3.106014 & 2.971355 & 0.000000 \\ \mathrm{H} & -360767 & -0.666502 & 0.000000 \\ & & & 0.0000000\end{array}$




\section{Protonation state: WSG}

\section{CASSCF}

$\begin{array}{ll}\mathrm{S}_{0} & -1059.83872688 \\ { }^{1} \pi \pi^{*} & -1059.68763260 \\ { }^{1} \pi \sigma^{*} & -1059.69440330\end{array}$

$\begin{array}{cc}\text { CASPT2 } & \\ \mathrm{S}_{0} & -1061.8581790213 \\ { }^{1} \pi \pi^{*} & -1061.7674185193 \\ { }^{1} \pi \sigma^{*} & -1061.7297159189\end{array}$

$\begin{array}{lrrr}\mathrm{C} & 3.401071 & -2.493767 & 0.000000 \\ \mathrm{~N} & 2.659643 & -1.435529 & 0.000000 \\ \mathrm{~N} & 4.765004 & -2.231487 & 0.000000 \\ \mathrm{C} & 4.948224 & -0.837506 & 0.000000 \\ \mathrm{O} & 6.026506 & -0.254384 & 0.000000 \\ \mathrm{C} & 3.546774 & -0.346870 & 0.000000 \\ \mathrm{C} & 3.225732 & 0.978550 & 0.000000 \\ \mathrm{H} & 4.092698 & 1.639403 & 0.000000 \\ \mathrm{C} & 1.937697 & 1.625338 & 0.000000 \\ \mathrm{C} & 0.699410 & 0.933273 & 0.000000 \\ \mathrm{H} & 0.693209 & -0.151002 & 0.000000 \\ \mathrm{C} & 1.899420 & 3.039900 & 0.000000 \\ \mathrm{H} & 2.837071 & 3.591978 & 0.000000 \\ \mathrm{C} & -0.505236 & 1.618930 & 0.000000 \\ \mathrm{H} & -1.434594 & 1.055881 & 0.000000 \\ \mathrm{C} & 0.701924 & 3.732399 & 0.000000 \\ \mathrm{H} & 0.674917 & 4.817722 & 0.000000 \\ \mathrm{C} & -0.517251 & 3.028617 & 0.000000 \\ \mathrm{H} & 5.518212 & -2.900691 & 0.000000 \\ \mathrm{H} & -6.531528 & -0.161635 & 0.890577 \\ \mathrm{C} & -5.978338 & -0.499362 & 0.000000 \\ \mathrm{H} & -5.988222 & -1.596266 & 0.000000 \\ \mathrm{H} & -6.531528 & -0.161635 & -0.890577 \\ \mathrm{H} & -4.429115 & -3.509467 & 0.883744 \\ \mathrm{C} & -3.834055 & -3.768403 & 0.000000 \\ \mathrm{H} & -3.642524 & -4.843563 & 0.000000 \\ \mathrm{H} & -4.429115 & -3.509467 & -0.883744 \\ \mathrm{C} & -2.509403 & -2.974538 & 0.000000 \\ \mathrm{H} & 3.018526 & -3.508429 & 0.000000 \\ \mathrm{O} & -1.650614 & 3.758866 & 0.000000 \\ \mathrm{O} & -3.985516 & 2.495460 & 0.000000 \\ \mathrm{O} & -4.662740 & -0.001740 & 0.000000 \\ \mathrm{O} & -2.622521 & -1.692895 & 0.000000 \\ \mathrm{O} & -1.442657 & -3.614099 & 0.000000 \\ \mathrm{H} & -3.106014 & 2.971355 & 0.000000 \\ & -4.401001 & 0.963399 & 0.000000 \\ -3.424493 & -1.028132 & 0.000000 \\ \mathrm{H} & -4.789926 & 3.022998 & 0.000000\end{array}$

